\section{Equivalência semântica e avaliação da consistência interna da versão em português do Sociocultural Attitudes Towards Appearance Questionnaire-3 (SATAQ-3)}

\author{
Semantic equivalence and internal consistency \\ of the Brazilian Portuguese version of the \\ Sociocultural Attitudes Towards Appearance \\ Questionnaire-3 (SATAQ-3)
}

Ana Carolina Soares Amaral 1

Táki Athanássios Cordás 2

Maria Aparecida Conti 2

Maria Elisa Caputo Ferreira 1
${ }_{1}^{1}$ Faculdade de Educação

Física e Desportos,

Universidade Federal de Juiz de Fora, Juiz de Fora, Brasil.

2 Ambulatório de Bulimia

e Transtornos Alimentares,

Universidade de São Paulo,

São Paulo, Brasil.

Correspondência

A. C. S. Amaral

Laboratório de Estudos

do Corpo, Faculdade de

Educação Física e Desportos,

Universidade Federal de Juiz

de Fora.

Rua José Lourenço Kelmer s/n, Campus Universitário, Juiz de Fora, MG 36036-900, Brasil. acsamaral@hotmail.com

\section{Abstract}

This study aimed to describe the cross-cultural adaptation of the Sociocultural Attitudes Towards Appearance Questionnaire-3 (SATAQ-3) into Brazilian Portuguese. The methodology involved the following stages: (1) translation of the questionnaire into Portuguese; (2) back-translation into English; (3) meeting with experts to prepare a draft version; (4) assessment of verbal understanding of the draft by experts and by a sample of the target population; and (5) analysis of the tool's internal consistency, using Cronbach's alpha. The questionnaire was translated into Portuguese, and the scale's final version included 30 items, as in the original. Both the experts and target population members assessed all the items as easy to understand. Internal consistency was satisfactory, reaching 0.91 for the scale as a whole. The questionnaire has now been translated and adapted into Portuguese, with evidence of clear understanding and internal consistency. However, it is still necessary to assess its measurement equivalence, external validity, and reproducibility.

Body Image; Eating Disorders; Translations; Validation Studies

\section{Introdução}

As pesquisas em relação à imagem corporal têm ocupado um lugar de destaque no cenário acadêmico, sendo um tema recorrente em discussões nas áreas de Psicologia, Sociologia, Medicina, Pedagogia, Educação Física, entre outras.

O conceito "imagem corporal" refere-se a um constructo composto, envolvendo, no mínimo, duas dimensões: a perceptiva - relacionada à estimativa do tamanho e da forma corporais, e a atitudinal - referente às crenças e aos comportamentos focados na aparência 1. Campana \& Tavares 2 caracterizam essa dimensão atitudinal da imagem corporal como sendo formada pelos componentes: insatisfação geral subjetiva - referente à (in)satisfação do sujeito em relação à sua aparência como um todo, componente afetivo - relacionado às emoções relativas à aparência física, componente cognitivo - referente ao investimento na aparência física e a pensamentos e crenças sobre o corpo, e o componente comportamental - relacionado a situações de evitação e checagem do corpo.

$\mathrm{O}$ contexto cultural gera grande influência sobre a imagem corporal, sendo destacado como uma das principais fontes das quais emanam diversas atitudes em relação ao corpo. Alguns autores destacam que a influência dos padrões de beleza difundidos pela mídia são aspectos relevantes no que se refere ao desenvolvimento e à manutenção de transtornos alimentares e de 
imagem ${ }^{3}$, bem como ao consumo de esteroides anabolizantes e à realização de cirurgias plásticas estéticas 4,5. Como a prevalência desses transtornos e práticas tem se tornado cada vez mais alta entre a população brasileira $4,5,6,7$, faz-se necessária a avaliação dos diversos componentes da imagem corporal, a fim de investigar o papel de suas dimensões no desenvolvimento desses comportamentos, que chegam a se configurar como problema de saúde pública 6 .

Por ser um constructo multidimensional, existem diversas formas de se avaliar os componentes da imagem corporal. As escalas e os questionários autoavaliativos são os principais instrumentos utilizados no acesso a esse constructo, provavelmente por sua praticidade de aplicação e análise.

Entre os componentes da imagem corporal, o cognitivo consiste naquele com um menor número de instrumentos disponíveis validados para a população brasileira. Ressalta-se que uma das escalas destinadas à investigação das crenças e dos pensamentos sobre o corpo é o Sociocultural Attitudes Towards Appearance Questionnaire (SATAQ), desenvolvido originalmente

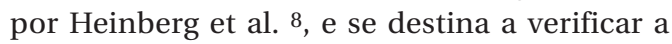
aceitação, por mulheres, dos padrões de aparência socialmente estabelecidos. Essa versão conta com 14 perguntas desenvolvidas para avaliar a internalização dos ideais de corpo divulgados pela mídia e a familiarização das mulheres com estes padrões.

A fim de avaliar outros aspectos dessa influência da mídia, Thompson et al. 9 desenvolveram o SATAQ-3, uma versão revisada sob a forma de questionário, em escala Likert de pontos, de 1 (Definitely Disagree) a 5 (Definitely agree). A versão original do instrumento foi validada para uma amostra de universitárias americanas, com idades entre 17 e 25 anos. Para a construção do SATAQ-3, foram utilizadas algumas das questões que compunham a primeira versão do SATAQ, acrescidas de itens que indicam a influência da mídia em relação aos esportes e ao exercício ${ }^{9}$. O escore total do SATAQ-3 é calculado pela soma das respostas, sendo que a maior pontuação representa maior influência dos aspectos socioculturais na imagem corporal do indivíduo. O questionário é composto por 30 perguntas destinadas a avaliar a internalização geral dos padrões socialmente estabelecidos (9 itens), incluindo o ideal de corpo atlético (5 itens), a pressão exercida por estes padrões (7 itens) e a mídia como fonte de informações sobre aparência (9 itens). A consistência interna das subescalas no estudo original foi de 0,96 para internalização geral, 0,95 para internalização atlética, 0,92 para pressão e 0,96 para informação, sendo que o total da escala obteve um alfa de Cronbach de 0,96 . Os autores observaram, ainda, a validade discriminante da escala a partir da aplicação do SATAQ-3 a uma amostra de pacientes com transtornos alimentares, verificando que as pacientes com transtorno obtiveram escores significativamente maiores que os controles em todas as subescalas $(\mathrm{p}<0,017)$.

A internalização do padrão de corpo perfeito se torna um importante aspecto no que tange à imagem corporal por representar o aval ativo dos ideais de aparência, ou seja, a incorporação do valor ao ponto de modificar suas atitudes e comportamentos pessoais 10 .

Com o crescimento das pesquisas acerca da imagem corporal, muitos pesquisadores têm se dedicado à criação e/ou adaptação transcultural de escalas. Seguindo essa perspectiva, as duas versões principais do SATAQ têm sido traduzidas e validadas para diversas populações no mundo, o que reflete a grande importância que vem sendo dada por pesquisadores de diversos países a esse componente da imagem corporal $11,12,13,14,15,16$.

Entre as validações do SATAQ-3 para outros contextos, destaca-se a versão francesa 16, na qual os autores confirmaram, por meio de uma análise fatorial exploratória, os quatro constructos da escala original e valores do coeficiente alfa de Cronbach variando entre 0,82 e 0,92. Markland \& Oliver 13 realizaram uma análise fatorial confirmatória a fim de comprovar a validade de constructo do SATAQ-3 em uma amostra de universitárias britânicas, e verificaram os mesmos quatro fatores da versão original. Uma versão do SATAQ-3 para uma amostra de mulheres malaias 15 comprovou que o instrumento pode ser, também, utilizado em culturas não ocidentais. Nesse estudo, os autores atestaram a estrutura fatorial do SATAQ-3 na Malásia e verificaram coeficientes alfa de Cronbach satisfatórios, variando de 0,82 a 0,94 .

O SATAQ-3 tem sido utilizado em diversas investigações acerca da influência da mídia na imagem corporal, sendo o instrumento mais difundido na atualidade para o estudo do componente cognitivo ${ }^{15}$. Entretanto, esse questionário ainda não possui uma versão na língua portuguesa nem registros da avaliação de suas qualidades psicométricas para a realidade brasileira.

Esse instrumento se configura como uma possibilidade de acesso à dimensão cognitiva da imagem corporal e, em função da crescente influência da mídia na busca pelo corpo perfeito, a investigação acerca deste componente se faz de extrema relevância no estudo dos fatores relacionados aos transtornos de imagem e alimentares. Por ser autoavaliativo, o SATAQ-3 é de fácil 
aplicação em grandes amostras e, devido à sua grande aceitação no meio científico, uma versão na língua portuguesa possibilitará comparações futuras com outras populações por meio de estudos transculturais.

Assim, o presente trabalho objetiva descrever as etapas iniciais do processo de adaptação transcultural do SATAQ-3, que se desdobram em sua tradução, retrotradução, avaliação da equivalência semântica, compreensão verbal e a avaliação da consistência interna para a população brasileira.

\section{Métodos}

O processo de adaptação transcultural baseouse nos procedimentos sugeridos por Guillemin et al. 17 por serem largamente os mais utilizados pelos pesquisadores brasileiros na tradução de instrumentos na área da saúde. Buscou-se, igualmente, preservar a avaliação das equivalências proposta por Herdman et al. 18,19 e descrita em detalhes por Reichenheim \& Moraes 20. Sendo assim, o primeiro passo foi a solicitação de autorização aos autores das escalas originais feminina e masculina para seu manuseio, sendo estas concedidas nos dias 25 de março e 30 de março de 2010, respectivamente.

Segundo Beaton et al. ${ }^{21}$, a adaptação transcultural pode ser definida como o processo que examina a língua (tradução) e questões de adaptação no processo de preparar um questionário para uso em outro contexto cultural. Autores como Herdman et al. 18,19 destacam que esse processo garante que um instrumento possa ser utilizado em outras culturas, desde que haja uma equivalência entre as versões traduzidas de um mesmo questionário.

\section{Procedimentos}

Segundo Reichenheim \& Moraes 20, o primeiro passo para a realização da adaptação transcultural de um instrumento para outro contexto cultural é a avaliação de sua equivalência conceitual. Pesquisas recentes têm demonstrado a crescente prevalência de insatisfação corporal entre amostras brasileiras variadas 6,7 , indicando que a preocupação com o corpo é um fenômeno presente também na realidade brasileira.

A primeira etapa consistiu da tradução do instrumento, em suas versões masculina e feminina, da língua inglesa para o português, sendo que esta foi realizada por dois tradutores independentes e nativos brasileiros. Um desses tradutores possuía conhecimento sobre o fenômeno avaliado pelo instrumento, enquanto o outro desconhecia o tema. Em seguida, os dois tradutores propuseram uma síntese de suas traduções, a qual foi encaminhada para a retrotradução para dois tradutores nativos da língua inglesa que desconheciam o questionário original 17.

Na segunda etapa, procedeu-se a revisão do instrumento e a avaliação da equivalência semântica por um comitê de peritos constituído pela pesquisadora responsável, uma especialista em línguas, uma das tradutoras e uma especialista em metodologia de adaptação transcultural de escalas. A avaliação da equivalência semântica buscou manter os mesmos significados entre as palavras e expressões do instrumento original e do traduzido. A revisão a respeito do modo de administração e aplicação do instrumento traduzido também foi realizada, garantindo que, mesmo com a opção por alteração no formato da escala, o conteúdo fosse semelhante, assegurando a equivalência operacional. A partir desse processo, foi elaborada a primeira versão do instrumento.

Para avaliação da compreensão verbal, esta primeira versão foi apresentada a profissionais especialistas na área de transtornos alimentares (nutricionista, psicólogo, psiquiatra e educador físico). Ao especialista foi solicitado responder a uma escala numérica adaptada 22,23 , a fim de avaliar a clareza e a compreensão de cada questão e do instrumento na íntegra. O participante foi orientado a indicar o quanto compreendeu de cada item do instrumento, por meio de uma escala Likert de 0 (não entendi nada) a 5 (entendi perfeitamente e não tenho dúvidas). Conforme Conti et al. ${ }^{23}$, foi estabelecido que as respostas 0 , 1, 2 e 3 eram indicadoras de uma compreensão insuficiente. O especialista foi ainda orientado, caso não compreendesse a questão ou a linguagem não parecesse adequada, que sugerisse alterações justificando os motivos.

Essa mesma escala de compreensão verbal foi apresentada a 60 jovens, estudantes do curso de Educação Física, de uma instituição pública do Município de Juiz de Fora, Estado de Minas Gerais, sendo 30 homens e 30 mulheres, selecionados de forma aleatória simples e que concordaram em participar voluntariamente da pesquisa. Do mesmo modo que foi pedido aos especialistas, solicitou-se aos jovens que registrassem suas sugestões e dificuldades na compreensão das questões. A fim de se analisar os escores de compreensão verbal de cada uma das 30 perguntas que compõem o questionário, foram calculados a média e o desvio-padrão das respostas dos especialistas e dos universitários, de forma a se ter uma visão geral da compreensão verbal das questões. Uma nova versão do instrumento foi formulada a partir da observação dos dados apresentados pelos especialistas e pelos jovens entrevistados. 
Como última etapa, a versão final do instrumento foi aplicada a um grupo de 86 universitários, sendo 46 mulheres e 40 homens, para avaliar o grau de consistência interna das questões que fazem parte do instrumento, bem como de cada uma das quatro subescalas, por meio da análise do coeficiente alfa de Cronbach. Os voluntários foram selecionados de forma aleatória e assinaram o Termo de Consentimento Livre e Esclarecido. Ao questionário foram acrescentadas perguntas diretas que buscavam verificar dados demográficos dos participantes, como idade e sexo.

Para as análises estatísticas utilizou-se o programa SPSS versão 16.0 (SPSS Inc., Chicago, Estados Unidos). Foram realizadas análises descritivas (média e desvio-padrão) e inferencial (alfa de Cronbach).

O projeto do presente estudo foi aprovado pelo Comitê de Ética e Pesquisa da Universidade Federal de Juiz de Fora (UFJF) em 15 de julho de 2010 (parecer no. 148/2010), e sua execução está de acordo com as normas da Resolução no ${ }^{\circ}$. 196/96 do Conselho Nacional de Saúde.

\section{Resultados}

Participaram deste estudo nove profissionais especialistas na área de transtornos alimentares, sendo três nutricionistas, três psicólogos, dois psiquiatras e uma educadora física. A amostra da população-alvo foi composta por 146 universitários, sendo 70 homens (20,7 anos $\pm 3,5$ anos) e 76 mulheres (20,3 anos $\pm 2,2$ anos).

\section{Tradução}

O primeiro item que mereceu atenção referiu-se a uma das opções de resposta, pois a tradução literal da palavra mostly é "a maioria”, o que não expressa a intenção avaliativa do questionário. Assim, optou-se pela utilização da expressão "em grande parte" e, dessa forma, garantiu-se uma maior clareza do conteúdo expresso, adequando-o à língua portuguesa.

Já nas questões 1, 5, 9,13, 17, 21, 25, 28 e 29, optou-se pela introdução do vocábulo "como" à tradução da expressão being attractive, com o intuito de dar maior clareza ao significado pretendido no instrumento original. Dessa forma, conseguiu-se garantir, por meio dessa expressão, o sentido de que a mídia se configura como fonte de informações sobre as características e as maneiras de se tornar uma pessoa atraente.

Como o instrumento não determina um período ao qual o participante deve se remeter ao responder às questões, entendeu-se que cada item se refere a acontecimentos que ocorreram em algum momento da vida do sujeito, sem registro temporal específico. Assim, optou-se pelo tempo verbal presente em todo o questionário. Quanto à expressão I have felt pressure, o comitê optou por sua tradução em forma de locução verbal: "já me senti pressionado", sugerindo mais claramente esta intenção do instrumento.

$\mathrm{Na}$ língua inglesa, a utilização do verbo to look conjugado a um adjetivo (look pretty e look muscular) transmite a ideia de algo momentâneo. Por isso, optou-se pela tradução na forma do verbo "ficar", indicando a tendência de modificação da aparência.

Já em relação à expressão music videos, optou-se pela única palavra disponível no dicionário da Academia Brasileira de Letras ${ }^{24}$ referente a vídeos musicais apresentados na televisão. Dessa forma, a expressão foi traduzida para a palavra "videoclipe".

Após revisão do questionário em suas versões feminina e masculina, escolhemos por fundir as duas versões em uma única escala. Como a adaptação masculina proposta por Karazsia \& Crowther 25 apenas substituiu as palavras que faziam menção à magreza por referência à muscularidade, seguiu-se esta mesma lógica na padronização do presente estudo. Ademais, essa fusão se justifica pela facilidade de aplicação de um mesmo questionário às duas populações simultaneamente.

Para que a fusão dos questionários fosse realizada, foi necessário o retorno das frases negativas à versão masculina do instrumento, pois a adaptação para esta população na língua inglesa havia transformado os itens com escore reverso em afirmações.

As etapas de tradução, retrotradução e a versão do instrumento produzida pelo comitê de peritos podem ser observadas nas Tabelas $1 \mathrm{e}$ 2. No que diz respeito ao título do questionário, optou-se por utilizar sua tradução, mantendo, contudo, a sigla do instrumento original, para que o mesmo seja facilmente reconhecido na comunidade científica.

Em relação à equivalência instrumental, ou seja, à forma de administração do questionário, o comitê optou por sua alteração. Foi estabelecida a apresentação do SATAQ-3 na forma de uma tabela, na qual cada linha representa um item do questionário e cada coluna uma opção de resposta.

\section{Compreensão verbal}

Entre os especialistas, as questões do SATAQ-3 obtiveram graus de compreensão verbal superiores a 4,3 (valor máximo $=5$ ), demonstrando que 
Avaliação da equivalência semântica entre a versão original do Sociocultural Attitudes Towards Appearance Questionnaire-3 (SATAQ-3) e a síntese das traduções. Juiz de Fora, Minas Gerais, Brasil, 2010.

\section{Documento original}

Sociocultural Attitudes Towards Appearance Questionnaire-3

Please read each of the following items carefully and indicate the number that best reflects your agreement with the statement.

Definitely Disagree; Mostly Disagree; Neither agree nor disagree; Mostly

Agree; Definitely Agree

TV programs are an important source of information about fashion and

"being attractive."

I've felt pressure from TV or magazines to lose weight

I do not care if my body looks like the body of people who are on TV

I compare my body to the bodies of people who are on TV.

TV commercials are an important source of information about

fashion and "being attractive."

Female version: I do not feel pressure from TV or magazines to look pretty.

Male version: I've felt pressure from TV or magazines to look muscular.

I would like my body to look like the models who appear in magazines.

I compare my appearance to the appearance of TV and movie stars.

Music videos on TV are not an important source of information about

fashion and "being attractive."

Female Version: I've felt pressure from TV and magazines to be thin.

Male Version: I've felt pressure from TV and magazines to be muscular.

I would like my body to look like the people who are in movies.

I do not compare my body to the bodies of people who appear in magazines.

Magazine articles are not an important source of information about fashion and "being attractive."

I've felt pressure from TV or magazines to have a perfect body.

I wish I looked like the models in music videos.

I compare my appearance to the appearance of people in magazines.

Magazine advertisements are an important source of information about fashion and "being attractive."

I've felt pressure from TV or magazines to diet.

I do not wish to look as athletic as the people in magazines.

I compare my body to that of people in "good shape".

Pictures in magazines are an important source of information about fashion and "being attractive".

I've felt pressure from TV or magazines to exercise.

I wish I looked as athletic as sports stars.

I compare my body to that of people who are athletic.

\section{Síntese das traduções}

Questionário de Atitudes Socioculturais Relativas à Aparência-3 Por favor, leia cada um dos itens abaixo cuidadosamente e indique o número que melhor reflete o quanto você concorda com a afirmação. Discordo totalmente; Discordo parcialmente; Nem concordo, nem discordo; Concordo parcialmente; Concordo totalmente.

Programas de TV são uma fonte importante de informações sobre moda e sobre "como ser atraente".

Já senti pressão da TV ou de revistas para perder peso.

Não me importo se o meu corpo se parece com o de pessoas que estão na TV.

Comparo meu corpo com o de pessoas que estão na TV.

Comerciais de TV são uma fonte importante de informações sobre moda e sobre "como ser atraente".

Versão feminina: Não me sinto pressionada pela TV ou revistas para ser bonita.

Versão masculina: Já senti pressão da TV ou de revistas para parecer musculoso.

Gostaria que meu corpo se parecesse com o das modelos que aparecem nas revistas.

Comparo a minha aparência com a das estrelas de TV e do cinema.

Clips de música na TV não são uma fonte importante de informações sobre moda e sobre "como ser atraente".

Versão feminina: Já senti pressão da TV ou de revistas para ser magra.

Versão masculina: Já senti pressão da TV ou de revistas para ser musculoso.

Gostaria que meu corpo se parecesse com o das modelos que aparecem nos filmes.

Comparo meu corpo com o das pessoas que aparecem nas revistas.

Artigos de revistas não são uma fonte importante de informações sobre moda e sobre "como ser atraente".

Já senti pressão da TV ou de revistas para ter um corpo perfeito.

Gostaria de me parecer com as modelos dos clips de música.

Comparo a minha aparência com a das pessoas nas revistas.

Anúncios em revistas são uma fonte importante de informações sobre moda e sobre "como ser atraente".

Já senti pressão da TV ou de revistas para fazer dieta.

Não desejo ser tão atlética quanto as pessoas nas revistas.

Eu comparo o meu corpo ao de pessoas em "boa forma".

Fotos em revistas são uma fonte importante de informações sobre moda e sobre "como ser atraente".

Já senti pressão da TV ou de revistas para me exercitar.

Eu gostaria de ter uma aparência tão atlética quanto a das estrelas do esporte.

Eu comparo o meu corpo com o de pessoas que são atléticas.

(continua) 
Movies are an important source of information about fashion and "being attractive".

I've felt pressure from TV or magazines to change my appearance.

I do not try to look like the people on TV.

Movie starts are not an important source of information about fashion and "being attractive".

Famous people are an important source of information about fashion and "being attractive".

I try to look like sports athletes.
Filmes são uma fonte importante de informações sobre moda e sobre "como ser atraente".

Já senti pressão da TV ou de revistas para mudar minha aparência.

Não tento me parecer com as pessoas na TV.

Estrelas de cinema não são uma fonte importante de informações sobre moda e sobre "como ser atraente".

Pessoas famosas são uma fonte importante de informações sobre moda e sobre "como ser atraente".

Tento me parecer com atletas.

Tabela 2

Avaliação da equivalência semântica entre a versão retrotraduzida do Sociocultural Attitudes Towards Appearance Questionnaire-3 e a versão do Comitê de Peritos. Juiz de Fora, Minas Gerais, Brasil, 2010.

\section{Retrotradução}

Sociocultural Attitudes Towards Appearance Questionnaire-3

Please read each of the items below carefully and mark the number which best reflects the degree to which you agree with the statement.

Definitely disagree

Mostly disagree

Don't agree or disagree

Mostly agree

Definitely agree

TV programs are an important source of information on fashion and "how to be attractive".

I've felt pressure from TV or magazines to lose weight.

It doesn't matter to me if my body looks like those of people who are on TV.

I compare my body to those of people who are on TV.

TV commercials are an important source of information on fashion and on "how to be attractive".

Female Version: I don't feel pressured by TV or magazines to be beautiful. Male Version: I've felt pressure from TV or magazines to appear muscular.

I would like for my body to look like one of those of the models who appear in magazines.

I compare my appearance to that of movie and TV stars.

Music videos on TV are an important source of information about fashion and "how to be attractive".

Female Version: I've felt pressure from TV or magazines to be thin. Male Version: I've felt pressure from TV or magazines to be muscular.

I would like for my body to look like those of the standard body types which appear in movies.

I don't compare my body to those of people who appear in magazines.

\section{Versão do Comitê de Peritos}

Questionário de Atitudes Socioculturais em relação à aparência

Por favor, leia cada um dos itens abaixo cuidadosamente e indique o número que melhor reflete o quanto você concorda com a afirmação. Discordo totalmente $=1$

Discordo em grande parte $=2$

Nem concordo nem discordo $=3$

Concordo em grande parte $=4$

Concordo totalmente $=5$

1. Programas de TV são importantes fontes de informação sobre moda e sobre "como ser atraente".

2. Já me senti pressionado(a) pela TV ou pelas revistas para perder peso.

3. Não me importo se meu corpo se parece com o de pessoas que estão na TV.

4. Comparo meu corpo com o de pessoas que estão na TV.

5. Comerciais de TV são importantes fontes de informação sobre moda e sobre "como ser atraente".

6. PARA MULHERES: Não me sinto pressionada pela TV ou pelas revistas para ficar bonita.

PARA HOMENS: Não me sinto pressionado pela TV ou pelas revistas para ficar musculoso.

7. Gostaria que meu corpo fosse parecido com o dos (as) modelos das revistas.

8. Comparo minha aparência com a das estrelas de TV e do cinema.

9. Clipes de música não são importantes fontes de informação sobre moda e sobre "como ser atraente".

10. PARA MULHERES: Já me senti pressionada pela TV ou pelas revistas para ser magra.

PARA HOMENS: Já me senti pressionado pela TV ou pelas revistas para ser musculoso.

11. Gostaria que meu corpo fosse parecido com o dos (as) modelos dos filmes.

12. Não comparo meu corpo com os das pessoas das revistas.

(continua) 


\begin{tabular}{|c|c|}
\hline Retrotradução & Versão do Comitê de Peritos \\
\hline $\begin{array}{l}\text { Magazine articles are not an important source of information on fashion or } \\
\text { "how to be attractive". }\end{array}$ & $\begin{array}{l}\text { 13. Artigos de revistas não são importantes fontes de informação sobre } \\
\text { moda e sobre "como ser atraente". }\end{array}$ \\
\hline I've felt pressure from TV or magazines to have a perfect body. & $\begin{array}{l}\text { 14. Já me senti pressionado(a) pela TV ou pelas revistas para ter um corpo } \\
\text { perfeito. }\end{array}$ \\
\hline I would like to look like the models shown in music videos. & 15. Gostaria de me parecer com os(as) modelos dos clipes de música. \\
\hline I compare my appearance to that of people in magazines. & 16. Comparo minha aparência com a das pessoas das revistas. \\
\hline $\begin{array}{l}\text { Advertisements in magazines are an important source of information on } \\
\text { fashion and "how to be attractive". }\end{array}$ & $\begin{array}{l}\text { 17. Anúncios em revistas são importantes fontes de informação sobre } \\
\text { moda e sobre "como ser atraente". }\end{array}$ \\
\hline I've felt pressure from TV or magazines to go on a diet. & 18. Já me senti pressionado(a) pela TV ou pelas revistas para fazer dieta. \\
\hline I don't want to be as athletic as people in the magazines. & 19. Não desejo ser tão atlético(a) quanto as pessoas das revistas. \\
\hline I compare my body to those of people who are in "good shape". & 20. Comparo meu corpo ao das pessoas em boa forma. \\
\hline $\begin{array}{l}\text { Photos in magazines are an important source of information on fashion } \\
\text { and "how to be attractive". }\end{array}$ & $\begin{array}{l}\text { 21. Fotos de revistas são importantes fontes de informação sobre moda e } \\
\text { sobre "como ser atraente". }\end{array}$ \\
\hline I've felt pressure from TV or magazines to exercise. & $\begin{array}{l}\text { 22. Já me senti pressionado(a) pela TV ou pelas revistas para praticar } \\
\text { exercícios. }\end{array}$ \\
\hline I would like to have an appearance as athletic as that of sports stars. & $\begin{array}{l}\text { 23. Gostaria de ter uma aparência tão atlética quanto a das estrelas do } \\
\text { esporte. }\end{array}$ \\
\hline I compare my body to those of athletic people. & 24. Comparo meu corpo com o de pessoas atléticas. \\
\hline Movies are an important source of information on fashion and & 25. Filmes são importantes fontes de informação sobre moda e sobre \\
\hline "how to be attractive". & "como ser atraente". \\
\hline I've felt pressure from TV or magazines to change my appearance. & $\begin{array}{l}\text { 26. Já me senti pressionado(a) pela TV ou pelas revistas para mudar minha } \\
\text { aparência. }\end{array}$ \\
\hline I don't try to look like people on TV. & 27. Não tento me parecer com as pessoas da TV. \\
\hline $\begin{array}{l}\text { Movie stars are not an important source of information on fashion and } \\
\text { "how to be attractive". }\end{array}$ & $\begin{array}{l}\text { 28. Estrelas de cinema não são importantes fontes de informação sobre } \\
\text { moda e sobre "como ser atraente". }\end{array}$ \\
\hline $\begin{array}{l}\text { Famous people are an important source of information on fashion and } \\
\text { "how to be attractive". }\end{array}$ & $\begin{array}{l}\text { 29. Pessoas famosas são importantes fontes de informação sobre moda e } \\
\text { sobre "como ser atraente". }\end{array}$ \\
\hline I try to look like athletes. & 30. Tento me parecer com atletas. \\
\hline
\end{tabular}

as mesmas foram avaliadas como de fácil compreensão (Tabela 3).

Os jovens entrevistados nessa etapa destacaram a clareza das perguntas. Esse fato foi confirmado pelos graus médios de compreensão verbal, apresentados na Tabela 3, na qual percebe-se que todas as questões obtiveram valores médios superiores a 4,3 (valor máximo = 4,9).

As sugestões dadas pelos especialistas e pelos estudantes foram reportadas ao Comitê de Peritos que, com base na análise e no julgamento das mesmas, produziu a versão final do instrumento (Figura 1).

\section{Consistência interna}

O instrumento demonstrou valores do coeficiente alfa de Cronbach de 0,91 entre as mulheres e 0,92 entre os homens. Ao se considerarem os itens do questionário, independentemente do sexo, o valor do coeficiente foi de 0,91 .

Quando analisadas cada uma das quatro subescalas do instrumento, os valores encontrados foram de 0,90 para internalização geral (sendo 0,88 entre as mulheres e 0,90 entre os homens), 0,81 para a subescala internalização atlética $(0,74$ para as mulheres e 0,86 para os homens), 0,85 para a subescala pressão $(0,87$ e 0,82 para mulheres e homens, respectivamente) e 0,82 para informação (0,83 entre as mulheres e 0,82 entre os homens).

\section{Discussão}

As pesquisas que buscam investigar a imagem corporal e seus componentes têm se tornado cada vez mais presentes no meio científico. Thompson 26 destaca a importância da escolha de instrumentos adequados para a avaliação dos componentes da imagem corporal, sugerindo, também, que estas escalas possuam validade e fidedignidade comprovadas. O SATAQ-3 se destina a avaliar o componente cognitivo da imagem corporal, ainda carente de instrumentação avaliativa no Brasil. 
Tabela 3

Avaliação da compreensão verbal do Sociocultural Attitudes Towards Appearance Questionnaire-3 (SATAQ-3). Juiz de Fora, Minas Gerais, Brasil, 2010.

\begin{tabular}{|c|c|c|}
\hline Questões & $\begin{array}{l}\text { Especialistas } \\
\text { Média } \\
\text { (desvio-padrão) }\end{array}$ & $\begin{array}{c}\text { População-alvo } \\
\text { Média } \\
\text { (desvio-padrão) }\end{array}$ \\
\hline 1 & $5,00(0,00)$ & $4,83(0,42)$ \\
\hline 2 & $4,62(0,74)$ & $4,67(0,91)$ \\
\hline 3 & $4,75(0,71)$ & $4,63(0,94)$ \\
\hline 4 & $4,75(0,71)$ & $4,87(0,47)$ \\
\hline 5 & $4,88(0,35)$ & $4,90(0,40)$ \\
\hline 6 & $5,00(0,00)$ & $4,75(0,65)$ \\
\hline 7 & $4,88(0,35)$ & $4,93(0,41)$ \\
\hline 8 & $4,88(0,35)$ & $4,92(0,28)$ \\
\hline 9 & $4,88(0,35)$ & $4,55(0,85)$ \\
\hline 10 & $4,88(0,35)$ & $4,90(0,35)$ \\
\hline 11 & $4,75(0,71)$ & $4,88(0,37)$ \\
\hline 12 & $4,62(0,74)$ & $4,90(0,40)$ \\
\hline 13 & $5,00(0,00)$ & $4,68(0,72)$ \\
\hline 14 & $4,50(0,75)$ & $4,87(0,57)$ \\
\hline 15 & $5,00(0,00)$ & $4,82(0,57)$ \\
\hline 16 & $4,88(0,35)$ & $4,83(0,72)$ \\
\hline 17 & $5,00(0,00)$ & $4,82(0,62)$ \\
\hline 18 & $4,75(0,46)$ & $4,92(0,33)$ \\
\hline 19 & $4,62(0,74)$ & $4,72(0,64)$ \\
\hline 20 & $5,00(0,00)$ & $4,72(0,83)$ \\
\hline 21 & $4,88(0,35)$ & $4,77(0,62)$ \\
\hline 22 & $4,38(0,74)$ & $4,97(0,18)$ \\
\hline 23 & $4,88(0,35)$ & $4,77(0,65)$ \\
\hline 24 & $5,00(0,00)$ & $4,83(0,61)$ \\
\hline 25 & $4,86(0,38)$ & $4,73(0,69)$ \\
\hline 26 & $4,38(0,74)$ & $4,85(0,61)$ \\
\hline 27 & $4,75(0,46)$ & $4,77(0,79)$ \\
\hline 28 & $4,88(0,35)$ & $4,88(0,37)$ \\
\hline 29 & $4,88(0,35)$ & $4,90(0,35)$ \\
\hline 30 & $4,75(0,46)$ & $4,73(0,71)$ \\
\hline Total & $4,82(0,37)$ & $4,81(0,38)$ \\
\hline
\end{tabular}

Apesar de não existir um guia padrão-ouro para a realização das traduções, adaptações culturais e validações de instrumentos para a população brasileira, os guias existentes são unânimes ao defender a importância desse processo no desenvolvimento de escalas válidas e fidedignas 17,21. Os autores destacam que somente um processo de adaptação transcultural de qualidade é capaz de tornar um instrumento apto a ser utilizado em outro contexto cultural 18,19.

Com base nesses pressupostos, este estudo desenvolveu a tradução e adaptação trans- cultural do SATAQ-3 para a língua portuguesa, apresentando resultados satisfatórios. Incluiu a elaboração de três versões do instrumento, com a participação de peritos, especialistas da área de transtorno alimentar e população-alvo, para chegar a sua versão final. Comprovou, também, sua consistência interna, apresentado resultados promissores, com o valor alfa de Cronbach superior a 0,91 .

Em relação à sua apresentação, escolhemos por fundir as escalas feminina e masculina em um único questionário, a fim de facilitar a sua aplicação. Essa estratégia também foi utilizada em outras validações do SATAQ para outras culturas e populações 14,27, e não causou nenhum prejuízo ao entendimento dos itens, conforme pode ser observado pelos valores da consistência interna.

Ademais, não foram necessárias exclusões de itens da escala, e as alterações realizadas em relação ao instrumento original se justificaram pelo melhor entendimento dos enunciados. Essa medida está de acordo com o que propõem van Widenfelt et al. 28 , segundo os quais os tradutores devem avaliar criticamente o instrumento e notar os itens que não podem ser traduzidos de forma literal. Os autores destacam que a escolha pela tradução do enunciado deve ter como objetivo capturar a intenção do instrumento original, mesmo que, para isso, algumas palavras necessitem ser alteradas. Esse cuidado foi adotado neste estudo.

Já em relação à compreensão dos itens, sua avaliação foi satisfatória, com valores médios na escala de compreensão verbal, tanto por especialistas quanto pela população-alvo, superiores a 4,3 . Vale destacar que os mesmos atendem ao estabelecido por Conti et al. 23 , para os quais esse valor deve ser superior a 3 , para que sejam considerados de compreensão suficiente.

Interessante observar que, no estudo original para mulheres 9 , a consistência interna foi de 0,96 para toda a escala, valor muito próximo ao encontrado no presente trabalho. Na adaptação masculina 25 , os valores obtidos para o alfa de Cronbach também foram semelhantes. Quando analisadas cada uma das quatro subescalas que compõem o instrumento original, os valores de consistência interna também foram satisfatórios, variando de 0,74 a 0,90. O fato de os coeficientes alfa de Cronbach terem sido altos podem ser discutidos, também, em função do número de questões do instrumento (30), o qual pode ter colaborado para os valores encontrados. Apesar disso, deve-se ressaltar a importância desses resultados, que apontam para a fidedignidade do instrumento pela avaliação da consistência interna de todas as suas questões e de suas subescalas. 
Figura 1

Questionário de Atitudes Socioculturais em Relação à Aparência (SATAQ-3).

Por favor, leia cada um dos itens abaixo cuidadosamente e indique o número que melhor reflete o quanto você concorda com a afirmação.

Discordo totalmente $=1$

Discordo em grande parte $=2$

Nem concordo nem discordo $=3$

Concordo em grande parte $=4$

Concordo totalmente $=5$

\begin{tabular}{|c|c|c|c|c|c|c|}
\hline \multicolumn{2}{|r|}{ Pergunta } & 1 & 2 & 3 & 4 & 5 \\
\hline 1 & Programas de TV são importantes fontes de informação sobre moda e sobre "como ser atraente" & & & & & \\
\hline 2 & Já me senti pressionado(a) pela TV ou por revistas a perder peso & & & & & \\
\hline 3 & Não me importo se meu corpo se parece com o de pessoas que estão na TV & & & & & \\
\hline 4 & Comparo meu corpo com o de pessoas que estão na TV & & & & & \\
\hline 5 & Comerciais de TV são importantes fontes de informação sobre moda e sobre "como ser atraente" & & & & & \\
\hline 6 & $\begin{array}{l}\text { PARA MULHERES: Não me sinto pressionada pela TV ou pelas revistas a ficar bonita } \\
\text { PARA HOMENS: Não me sinto pressionado pela TV ou pelas revistas a ficar musculoso }\end{array}$ & & & & & \\
\hline 7 & Gostaria que meu corpo fosse parecido com o dos(as) modelos das revistas & & & & & \\
\hline 8 & Comparo minha aparência com a das estrelas de TV e do cinema & & & & & \\
\hline 9 & Videoclipes não são importantes fontes de informação sobre moda e sobre "como ser atraente" & & & & & \\
\hline 10 & $\begin{array}{l}\text { PARA MULHERES: Já me senti pressionada pela TV ou pelas revistas a ser magra } \\
\text { PARA HOMENS: Já me senti pressionado pela TV ou pelas revistas a ser musculoso }\end{array}$ & & & & & \\
\hline 11 & Gostaria que meu corpo fosse parecido com o dos(as) modelos dos filmes & & & & & \\
\hline 12 & Não comparo meu corpo com o das pessoas das revistas & & & & & \\
\hline 13 & Artigos de revistas não são importantes fontes de informação sobre moda e sobre "como ser atraente" & & & & & \\
\hline 14 & Já me senti pressionado(a) pela TV ou pelas revistas a ter um corpo perfeito & & & & & \\
\hline 15 & Gostaria de me parecer com os(as) modelos dos videoclipes & & & & & \\
\hline 16 & Comparo minha aparência com a das pessoas das revistas & & & & & \\
\hline 17 & Anúncios em revistas são importantes fontes de informação sobre moda e sobre "como ser atraente" & & & & & \\
\hline 18 & Já me senti pressionado(a) pela TV ou por revistas a fazer dieta & & & & & \\
\hline 19 & Não desejo ser tão atlético(a) quanto as pessoas das revistas & & & & & \\
\hline 20 & Comparo meu corpo ao das pessoas em boa forma & & & & & \\
\hline 21 & Fotos de revistas são importantes fontes de informação sobre moda e sobre "como ser atraente" & & & & & \\
\hline 22 & Já me senti pressionado(a) pela TV ou pelas revistas a praticar exercícios & & & & & \\
\hline 23 & Gostaria de ter uma aparência tão atlética quanto a das estrelas do esporte & & & & & \\
\hline 24 & Comparo meu corpo com o de pessoas atléticas & & & & & \\
\hline 25 & Filmes são importantes fontes de informação sobre moda e sobre "como ser atraente" & & & & & \\
\hline 26 & Já me senti pressionado(a) pela TV ou pelas revistas a mudar minha aparência & & & & & \\
\hline 27 & Não tento me parecer com as pessoas da TV & & & & & \\
\hline 28 & $\begin{array}{l}\text { Estrelas de cinema não são importantes fontes de informação sobre moda e sobre "como ser } \\
\text { atraente" }\end{array}$ & & & & & \\
\hline 29 & Pessoas famosas são importantes fontes de informação sobre moda e sobre "como ser atraente" & & & & & \\
\hline 30 & Tento me parecer com atletas & & & & & \\
\hline
\end{tabular}

É importante enfatizar a relevância clínica de instrumentos de avaliação dos componentes da imagem corporal, visto que distúrbios neste constructo são considerados fundamentais na etiologia dos transtornos alimentares 29. Estudos recentes destacam a crescente prevalência desses transtornos na população brasileira, o que aumenta ainda mais a necessidade de instrumentos válidos e confiáveis para a investigação dos aspectos precursores dos mesmos 6,7.
Dessa forma, como o presente estudo se limitou a descrever as etapas iniciais do processo de tradução, adaptação transcultural e avaliação da consistência interna do SATAQ-3 brasileiro, faz-se ainda necessária a avaliação de sua equivalência operacional e de mensuração, bem como de sua estabilidade, por meio do teste-reteste, a fim de comprovar suas condições psicométricas. Após essa confirmação, o Questionário de Atitudes Socioculturais em Relação à Aparên- 
cia (SATAQ-3) estará apto para a utilização em pesquisas com a população brasileira jovem e, também, para a sua adaptação a fim de que seja utilizado com outras populações, tais como indivíduos com transtornos alimentares, adolescentes, entre outros.

\section{Resumo}

O objetivo do estudo foi descrever o processo de adaptação transcultural do Sociocultural Attitudes Towards Appearance Questionnaire-3 (SATAQ-3) para a língua portuguesa. A metodologia foi baseada nas etapas de (1) tradução do questionário para o português; (2) retrotradução para o inglês; (3) comitê de peritos para construção da primeira versão; (4) avaliação da compreensão verbal por especialistas e por uma amostra da população-alvo; (5) análise da consistência interna do instrumento a partir do alfa de Cronbach. O instrumento foi traduzido para o português e a versão final contou com os 30 itens do instrumento original. Todos os itens foram interpretados como de fácil compreensão, tanto por especialistas quanto pela população-alvo. Os valores de consistência interna foram satisfatórios, sendo de 0,91 para toda a escala. $O$ instrumento encontra-se traduzido e adaptado para o português, com evidências de boa compreensão e consistência interna, sendo ainda necessária a avaliação de sua equivalência de mensuração, validade externa e reprodutibilidade.

Imagem Corporal; Transtornos Alimentares; Tradução; Estudos de Validação

\section{Colaboradores}

A. C. S. Amaral participou da elaboração do projeto, coleta e análise dos dados, redação e discussão do artigo. T. A. Cordás e M. A. Conti contribuíram na análise dos dados, na redação e revisão do artigo. M. E. C. Ferreira colaborou na coleta dos dados, na redação e revisão do artigo. 


\section{Referências}

1. Cash T, Pruzinsky T. Body image: a handbook of theory, research and clinical practice. New York: Guilford; 2002.

2. Campana ANNB, Tavares MCGCF. Avaliação da imagem corporal: instrumentos e diretrizes para pesquisa. São Paulo: Phorte Editora; 2009.

3. Stice E, Tristan J. Sociocultural preassures and body image disturbance: a comment on Cafri, Yamamiya, Brannick, and Thompson. Clin Psychol Sci Pract 2005; 12:443-6.

4. Iriart JAB, Chaves JC, Orleans RG. Culto ao corpo e uso de anabolizantes entre praticantes de musculação. Cad Saúde Pública 2009; 25:773-82.

5. Sarwer DB, Crerand CE. Body image and cosmetic medical treatments. Body Image 2004; 1:99-111.

6. Nunes MA, Barros FC, Olinto MTA, Camey S, Mari JD. Prevalence of abnormal eating behaviours and inappropriate methods of weight control in young women from Brazil: a population-based study. Eat Weight Disord 2003; 8:100-6.

7. Alves E, Vasconcelos FAG, Calvo MCM, Neves J. Prevalência de sintomas de anorexia nervosa e insatisfação com a imagem corporal em adolescentes do sexo feminino do Município de Florianópolis, Santa Catarina, Brasil. Cad Saúde Pública 2008; 24:503-12.

8. Heinberg LJ, Thompson JK, Stormer S. Development and validation of the Sociocultural Attitudes Towards Appearance Questionnaire. Int J Eat Disord 1995; 17:81-9.

9. Thompson JK, van den Berg P, Roehrig M, Guarda AS, Heinberg LJ. The Sociocultural Attitudes Towards Appearance Scale-3 (SATAQ-3): development and validation. Int J Eat Disord 2004; 35: 293-304.

10. Cafri G, Yamamyia Y, Brannick M, Thompson JK. The influence of Sociocultural Factors on Body Image: a meta-analysis. Clin Psychol Sci Pract 2005; 12:421-33

11. Bagnara S, Huon G, Donazzolo S. Factorial structure of the Sociocultural Attitudes Towards Appearance Questionnaire among Italian and Australian girls. Eur Eat Disord Rev 2004; 12:321-6.

12. Calogero RM, Davis WN, Thompson JK. The Sociocultural Attitudes Towards Appearance Quationnaire (SATAQ-3): reliability and normative comparisons of eating disordered patients. Body Image 2004; 1:193-8.

13. Markland D. Oliver EJ. The Sociocultural Attitudes Questionnaire-3: a confirmatory factor analysis. Body Image 2008; 5:116-21.

14. Knauss C, Paxton SJ, Alsaker FD. Validation of the german version of the Sociocultural Attitudes Towards Appearance Questionnaire (SATAQ-G). Body Image 2009; 6:113-20.

15. Swami V. An examination of the factor structure of the Sociocultural Attitudes Towards Appearance Questionnaire-3 in Malaysia. Body Image 2009; 6:129-32.
16. Rousseau A, Valls M, Chabrol H. The Sociocultural Attitudes Towards Appearance Scale-3 (SATAQ-3): etude de validation de la version française. L'Encéphale 2010; 36:270-6.

17. Guillemin F, Bombardier C, Beaton D. Cross-cultural adaptation of health-related quality of life measures: literature review and proposed guidelines. J Clin Epidemiol 1993; 46:1417-32.

18. Herdman, M, Fox-Rushby J, Badia X. 'Equivalence' and the translation and adaptation of health-related quality of life questionnaires. Qual Life Res 1997; 6:237-47.

19. Herdman, M, Fox-Rushby J, Badia X. A model of equivalence in the cultural adaptation of HRQoL instruments: the universalist approach. Qual Life Res 1998; 7:323-35.

20. Reichenheim ME, Moraes CL. Operacionalização de adaptação transcultural de instrumentos de aferição usados em epidemiologia. Rev Saúde Pública 2007; 41:665-73.

21. Beaton DE, Bombardier C, Guillemin F, Ferraz MB. Guidelines for the process of cross-cultural adaptation of self-report measures. Spine 2000; 25:3186-91.

22. Conti MA, Slater B, Latorre MRDO. Validação e reprodutibilidade da Escala de Evaluación de Insatisfación Corporal para Adolescentes. Rev Saúde Pública 2009; 43:515-24.

23. Conti MA, Scagliusi F, Queiroz GKO, Hearst N, Cordas TA. Adaptação transcultural: tradução e validação de conteúdo para o idioma português do modelo da Tripartite Influence Scale de insatisfação corporal. Cad Saúde Pública 2010; 26:503-13.

24. Houaiss A, Villar MS. Dicionário Houaiss da língua portuguesa. Rio de Janeiro: Objetiva; 2009.

25. Karazsia BT, Crowther JH. Psychological and behavioral correlates of the SATAQ-3 with males. Body Image 2008; 5:109-15.

26. Thompson JK. The (mis)measurement of body image: ten strategies to improve assessment for applied and research purposes. Body Image 2004; 1:7-14.

27. Smolak L, Levine MP, Thompson JK. The use of the Sociocultural Attitudes Towards Appearance Questionnaire with middle school boys and girls. Int J Eat Disord 2001; 29:216-23.

28. van Widenfelt BM, Trefers PDA, Beurs E, Siebelink BM, Koudijs E. Translation and cross-cultural adaptation of assessment instruments used in psychological research with children and families. Clin Child Fam Psychol Rev 2005; 8:135-47.

29. Morgan CM, Vecchiatti IR, Negrão AB. Etiologia dos transtornos alimentares: aspectos biológicos, psicológicos e socioculturais. Rev Bras Psiquiatr 2002; 24:18-23.

Recebido em 13/Out/2010

Versão final reapresentada em 24/Mai/2011

Aprovado em 11/Jun/2011 